\title{
La labor de los cuerpos académicos en el programa educativo de Mantenimiento Industrial caso de estudio UTCV
}

\section{The labor of academic bodies on the Industrial Maintenance educational programme study case of UTCV}

\author{
ARIAS-PRIETO, María Isabel†, LARA-SALAZAR, Ignacio Zeferino*, RODRIGUEZ-LÓPEZ, Julio \\ Cesar y FERNÁNDEZ-VÁSQUEZ, Celia
}

Universidad Tecnológica del Centro de Veracruz, Departamento de Ingeniería en Mantenimiento Industrial, Av. Universidad No. 350, Carretera Federal Cuitláhuac-La Tinaja, Loc. Dos Caminos, Cuitláhuac, Veracruz, CP.94910

ID $1^{\text {er }}$ Autor: María Isabel, Arias-Prieto / ORC ID: 0000-002-3270-397X, Researcher ID Thomson: O-9701-2018, CVU CONACYT ID: 928668

ID $1^{\mathrm{er}}$ Coautor: Ignacio Zeferino, Lara-Salazar / ORC ID: 0000-0002-2283-3471, Researcher ID Thomson: O-7840-2018, CVU CONACYT ID: 927366

ID $2^{\text {do }}$ Coautor: Julio Cesar, Rodriguez-López / ORC ID: 0000-0002-8610-0234, Researcher ID Thomson: O-8171-2018, CVU CONACYT ID: 927470

ID $3{ }^{\text {er }}$ Coautor: Celia, Fernández-Vásquez / ORC ID: 0000-0002-0929-344X, Researcher ID Thomson: O-7858-2018, CVU CONACYT ID: 638159

\section{Resumen}

La integración de un cuerpo académico es sin duda un paso importante para profesionalización de la labor docente; el presente trabajo pretende describir la experiencia de los trabajos desarrollados por el cuerpo académico del área de Mantenimiento Industrial de la Universidad Tecnológica de Veracruz (UTCV), con miras en el desarrollo de proyectos tecnológicos y de impacto social, que bajo la dirección de la Maestra Celia Fernández Vásquez, se han tenido logros importantes como la participación destacada en el Premio Nacional del Emprendedor en su última edición 2017. En este sentido se comparte la metodología que se ha llevado a cabo para la captación y apoyo de proyectos innovadores, inclusive en la participación para la obtención de patentes; se evidencia la participación de la sociedad con los cuerpos académicos en la aplicación de proyectos, sin dejar a un lado la ardua labor de los jóvenes, que gracias a su talento y disposición han puesto (a) -sociedad, establecer la el vínculo antes mencionado y aterrizar la tecnología a la sociedad mexicana. Objetivos: Captar y desarrollar proyectos innovadores de impacto social y tecnológicos, con el apoyo de los cuerpos académicos, incitando la participación de los alumnos y miembros de otros cuerpos académicos, para favorecer el desarrollo de la sociedad. Metodología: Se realizó la búsqueda de proyectos tecnológicos y de impacto social bajo el esquema de proyecto integrador de la UTCV. Una vez detectados se inicia el desarrollo del proyecto bajo la dirección de especialistas en la materia y la vinculación entre cuerpos acáćmicos de diferentes especialistas en la maenia y la vinculación proce salvaguardar los modelos de utildad. Un paso inportante es la participación activa de la sociedad en la adquisición de la tecnología desarrollada por alumnos y el cuerpo académico, para ello se realizan talleres y se invita a las personas a demostraciones prácticas de los proyectos elaborados. Finalmente, se realiza la participación en convocatorias para poder dar a conocer los proyectos y de esta manera contribuir en el proceso de investigación. Contribución: La tarea del cuerpo académico, ha permitido llevar a la sociedad el acceso a nuevas máquinas, herramientas y métodos, para mejorar sus condiciones de vida y hacer un uso adecuado de los recursos de los cuales dispone. Con los proyectos realizados se han aprovechado y reutilizado materiales para evitar la contaminación, favoreciendo el cuidado del medioambiente.

Cuerpo Académico, Proyectos, Sociedad

\section{Abstract}

The integration of academic bodies is an important step to the professionalization of the teacher's work. This article aims to describe the developed works by the CA3 of the Universidad Tecnológica del Centro de Veracruz, with a view to on the development of social and technology projects. With the supervision of the master development of social and technology projects. With the supervision of the master
Celia Fernández Vásquez important achievements have been obtained like the Celia Fernández Vásquez important achievements have been obtained like the
participation in the National Entrepreneur price in 2017. In this way the methodology is shared to the catchment and support of innovative projects including the patent process. It shows the participation of the society with the academic bodies in the implementation of projects without mentioning the effort of the students. All of the above favors the binomial: university-society, that under the perspective of the authors, the academic bodies are in charge of making this linkage and offer technologies closer to the Mexican society Objective: Capture and devep in her members of academic bodies to the society's develop. Methodology: The search of projects under the concept of integrated project, model of the UTCV, was carried out. Then the develop of the project starts with the help of experts and members of others academic bodies from different areas, during this time is necessary to patent the utility models. An important step is the active social participation in the acquisition of technology developed by students and academic bodies. For this purpose, the academic body offers workshops and gives practice demonstrations of practical projects. Finally, the academic boty participates on calls to show the recent reserch prects. Fint research and thereby contibuting to the research process. Contribution: The task academic allows to the society the accessing to new machines, tools and methods to improve its life condition and make good use of resources. With the projects the materials have been recycle to avoid the pollution in favor of the environment.

Citación: ARIAS-PRIETO, María Isabel, LARA-SALAZAR, Ignacio Zeferino, RODRIGUEZ-LÓPEZ, Julio Cesar y FERNÁNDEZVÁSQUEZ, Celia. La labor de los cuerpos académicos en el programa educativo de Mantenimiento Industrial caso de estudio UTCV. Revista de Gestión Universitaria. 2019. 3-8: 25-33

\footnotetext{
* Correspondencia del Autor (correo electrónico: Ignacio.lara@utcv.edu.mx)

$\dagger$ Investigador contribuyendo como primer autor.
} 


\section{Introducción}

La creación de los cuerpos académicos ha favorecido evidentemente el funcionamiento integral de las universidades, se genera una dinámica profesional que proyecta a las instituciones educativas a la generación de nuevos conocimientos y la vinculación de la universidad con la sociedad, generando propuestas para los problemas sociales y educativos (Beltrán, Sevilla, Martín, 2018). El cuerpo académico permite formarse por especialistas en diferentes áreas y al realizar esto, los proyectos generan un mayor alcance y con la contribución de los estudiantes se pueden generar modelos de impacto social.

Con ello se busca reflexionar sobre la experiencia del Cuerpo Académico de Gestión de Calidad y Eficiencia Industrial de la Universidad Tecnológica del Centro de Veracruz (UTCV) en la conformación de un nuevo cuerpo académico, los retos que le esperan para el desarrollo de producción académica como lo son desarrollo de proyectos, patentamiento de prototipos, participación con otros cuerpos académicos o grupos de investigación, la participación activa con la sociedad y demás actividades del quehacer del cuerpo académico, surgiendo a partir de esto, los siguientes cuestionamientos: ¿cuál ha sido el avance de la academia de Gestión de Calidad y Eficiencia Industrial desde su conformación? ¿cuáles son los retos que se deben superar para el cuerpo académico dé sus primeros pasos en la labor investigativa y social? ¿cuáles son los elementos que han permitido el desarrollo de proyectos de impacto social? ¿cómo proseguir en el fortalecimiento del cuerpo académico?

Por otra parte, se debe recalcar que las sociedades modernas demandan de manera urgente atención, en los últimos años diversas campañas se han promovido por parte de grandes empresas hacia la sociedad, tal es el caso de la utilización del plástico y el fomento del reciclado del mismo, estas acciones que hace años no se veían, hoy en día parecen obligarnos a reflexionar sobre lo que está ocurriendo. Derivado de esto y desde el punto de vista de los autores la labor académica debe trascender para el beneficio de la sociedad, entonces los proyectos y modelos de utilidad, aunque funcionen perfectamente en las industrias, deberían acercarse a las personas con la finalidad de resolver problemas cotidianos.
En el texto se exponen las experiencias y el proceso que se ha seguido desde la conformación del cuerpo académico de Gestión de Calidad y Eficiencia Industrial de la institución antes mencionada, dando como resultado la participación en concursos destacados y convocatorias nacionales. Siendo el caso más sobresaliente el haber obtenido un lugar dentro de los primeros cuatro finalistas en el premio nacional del emprendedor, promovido por el gobierno de la República Mexicana en el año 2017. En este sentido se ha trabajo de manera continua, a pesar de ser un cuerpo académico en proceso de registro, se desea demostrar que se puede llegar lejos y lo más importante ha sido aportar algo a la gente y los estudiantes, facilitándoles las herramientas necesarias y fomentando una conciencia del uso adecuado de los recursos de los cuales se disponen. La captación, seguimiento y desarrollo de proyectos integradores permite al cuerpo académico dar un paso importante en la profesionalización del docente. Por otro lado, en México las Universidades Tecnológicas han cumplido con el compromiso de formar estudiantes bajo el enfoque de competencias que se traducen en tareas profesionales, lo que les permite enfrentarse a nuevas situaciones; a nuevos retos (Flores, 2019).

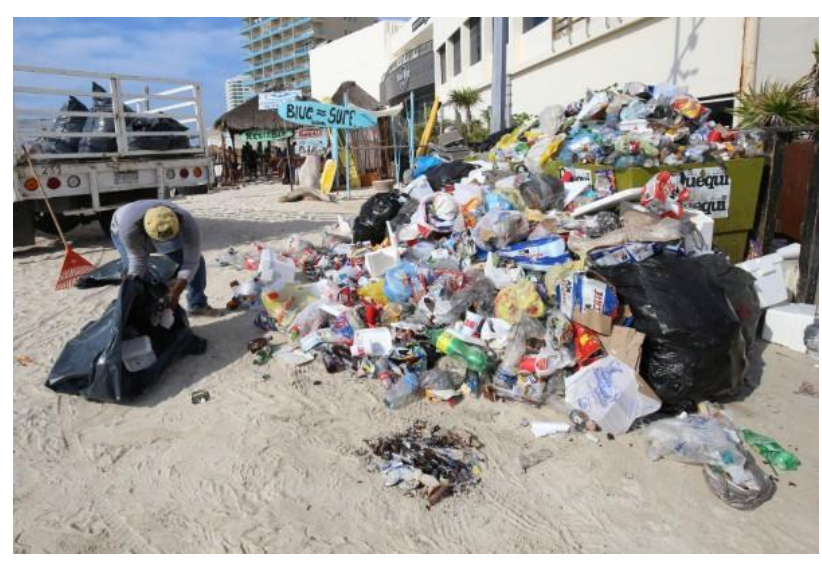

Figura 1 Generación de desechos plásticos Fuente: Heraldo de México

Con todo esto se espera dar un panorama general del camino que los cuerpos académicos necesitan tomar en la actualidad: apoyar y generar ideas, gestionar recursos en pro del medioambiente, buscar la generación de empleos y el surgimiento de nuevas empresas, investigar y enfocar los resultados hacia la gente, hacer partícipe a la sociedad y darle la importancia que debería tener, demostrar que labor académica puede resolver los problemas actuales. 


\section{Metodología a desarrollar}

\section{Los proyectos integradores}

Dentro de los diferentes programas educativos pertenecientes a las universidades tecnológicas y en especial la Universidad Tecnológica del Centro de Veracruz (UTCV), una de las formas de evaluar el aprendizaje significativo de los alumnos es mediante la presentación de un proyecto integrador cada final de cuatrimestre. El objetivo de esta actividad final, es de que el alumno integre los conocimientos adquiridos de sus asignaturas y lograr con ello la solución de diversas problemáticas sociales y de la industria.

Según Acevedo (2016) el proyecto integrador debe:

Unificar criterios de asignaturas específicas y transversales a través de un trabajo colaborativo.

- Evidenciar los resultados de aprendizaje donde apliquen de forma sinérgica los conocimientos adquiridos.

Motivar al estudiante a proponer soluciones de situaciones problemáticas enmarcadas en un contexto real durante todo su ciclo de formación.

El trabajo del cuerpo académico de Gestión de Calidad y Eficiencia Industrial ha sido desde un inicio participar activamente en el asesoramiento de proyectos y ha realizado un seguimiento a aquellos que por mucho tiempo no se tomaron en cuenta o necesitaban un realce. La tabla 1 muestra el número de proyectos integradores que se llevan a cabo en los cuatrimestres desde enero 2018 a agosto 2019.

\begin{tabular}{|l|r|l|r|}
\hline $\begin{array}{c}\text { Cuatrimestres } \\
\text { 2018 }\end{array}$ & \multicolumn{1}{c|}{ Total } & $\begin{array}{c}\text { Cuatrimestres } \\
\mathbf{2 0 1 9}\end{array}$ & Total \\
\hline Enero-abril & 78 & Enero-abril & 69 \\
\hline Mayo-agosto & 75 & Mayo agosto & 55 \\
\hline $\begin{array}{l}\text { Septiembre- } \\
\text { diciembre }\end{array}$ & 119 & & \\
\hline
\end{tabular}

Tabla 1 Proyectos integradores del P.E. de Mantenimiento Área Industrial

Desde el inicio del año 2019 entre los cuerpos académicos se optó por darle continuidad a los proyectos durante cinco cuatrimestres, periodo de formación del técnico superior universitario, y cuatro cuatrimestres para el nivel de ingeniería.
Con esto se podrán obtener prototipos mejor diseñados y acabados. En la misma tabla se muestra un aumento de proyectos integradores en el cuatrimestre septiembrediciembre, debido a que es el inicio del ciclo escolar y los alumnos de primero empiezan a desarrollar ideas para sus prototipos.

\begin{tabular}{|l|l|r|}
\hline \multicolumn{1}{|c|}{ Criterio } & Puntos \\
\hline 1 & $\begin{array}{l}\text { El equipo inicia y finaliza la presentación de su } \\
\text { proyecto en el tiempo establecido. }\end{array}$ & $\mathbf{2}$ \\
\hline 2 & $\begin{array}{l}\text { Los integrantes del equipo visten adecuadamente y } \\
\text { de forma uniforme. }\end{array}$ & $\mathbf{2}$ \\
\hline 3 & $\begin{array}{l}\text { Las diapositivas tienen un excelente diseño, buena } \\
\text { distribución del texto, transición y animación, } \\
\text { imágenes y no contienen faltas de ortografía. }\end{array}$ & $\mathbf{4}$ \\
\hline 4 & $\begin{array}{l}\text { La presentación incluye un planteamiento del } \\
\text { problema de acuerdo a una situación actual; un } \\
\text { objetivo general, objetivos específicos y una } \\
\text { justificación válida. }\end{array}$ & $\mathbf{1 0}$ \\
\hline 5 & $\begin{array}{l}\text { Se incluyen cálculos técnicos, diagramas y/o } \\
\text { esquemas que facilitan la comprensión de los } \\
\text { alumnos para la realización del prototipo. }\end{array}$ & $\mathbf{1 0}$ \\
\hline 6 & $\begin{array}{l}\text { Se presenta el prototipo en un software CAD con sus } \\
\text { anotaciones y acotaciones correspondientes. }\end{array}$ & $\mathbf{1 0}$ \\
\hline 7 & $\begin{array}{l}\text { El equipo muestra la metodología que utilizó para la } \\
\text { realización del diseño y/o construcción del prototipo. }\end{array}$ & $\mathbf{1 5}$ \\
\hline 8 & $\begin{array}{l}\text { El prototipo funciona correctamente el día de la } \\
\text { presentación del proyecto. }\end{array}$ & $\mathbf{1 5}$ \\
\hline 9 & $\begin{array}{l}\text { El equipo incluye un estudio económico y/o } \\
\text { financiero valido de su proyecto. }\end{array}$ & $\mathbf{1 0}$ \\
\hline 10 & $\begin{array}{l}\text { Se incluye una evaluación de resultados, estudios y/o } \\
\text { implementación del prototipo y mediante gráficas o } \\
\text { herramientas de calidad. }\end{array}$ & $\mathbf{1 0}$ \\
\hline 11 & $\begin{array}{l}\text { El equipo presenta las conclusiones y/o mejoras de } \\
\text { su proyecto. }\end{array}$ & $\mathbf{5}$ \\
\hline 12 & $\begin{array}{l}\text { Los integrantes del equipo participan de forma } \\
\text { uniforme en la presentación del proyecto. }\end{array}$ & $\mathbf{2}$ \\
\hline 13 & $\begin{array}{l}\text { Los integrantes del equipo responden acertadamente } \\
\text { alas preguntas realizadas por el jurado. }\end{array}$ & $\mathbf{5}$ \\
\hline Calificación: \\
\hline
\end{tabular}

Tabla 2 Rúbrica para la presentación de proyectos integradores

La evaluación se realiza a través de los cuerpos académicos, los profesores de asignatura se organizan con los integrantes del cuerpo académico para compartir las calificaciones de los avances de proyecto $\mathrm{y}$ evaluación final. Anteriormente cada asesor de grupo era el responsable de la evaluación y calificación de un grupo, hoy en día todos los proyectos pasan por una revisión de los cuerpos académicos. La tabla 2 muestra la rúbrica para evaluar los proyectos integradores, el cual se realiza en presencia del cuerpo académico.

$\mathrm{Al}$ término de las evaluaciones el cuerpo académico analiza cuáles fueron los proyectos más destacados, los que están en una fase menos desarrollada y a los que les falta mayor información. En ese momento se determina cuáles son los prototipos que pueden participar en convocatorias y concursos regionales y nacionales. 


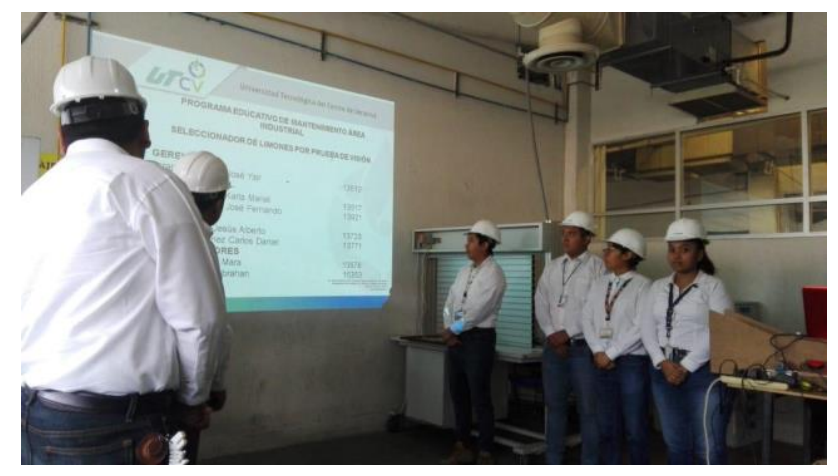

Figura 2 Presentación de proyectos al finalizar el cuatrimestre

Fuente: Elaboración Propia

\section{El proceso de patentamiento}

Una vez definido el o los proyectos a los que se les dará un seguimiento mayor, se pasa a un parte importante, que generalmente se omite, esta es patentar los modelos de utilidad y prototipos.

\section{Las Universidades Tecnológicas} dependen en gran medida del financiamiento gubernamental, por lo que deben ser capaces de financiar sus gastos a través de a la investigación y transferencia de tecnología. La mayoría de las universidades son instituciones docentes $\mathrm{y}$ formadoras, por lo que hay un escaso grado de interacción entre la investigación y las carencias del aparato productivo. "Entonces se vuelve necesaria la relación empresa-universidad para fortalecer las necesidades de la industria a través de los docentes y alumnos" (López, 2008, p.151).

En la UTCV se concibe la investigación como actividades internas que las realizan los cuerpos académicos y que van orientadas a la innovación y tecnología, lo exige el conocimiento de la inventiva, para la generación de prototipos, creados por los alumnos dentro de los cuatrimestres en curso, inclusive egresados. Los modelos y prototipos se deben de llevar al patentamiento, porque la mayoría de veces no se tiene el conocimiento para poder abordar este tema tan importante dentro de los grupos que conforman las academias en las diferentes especialidades. Una proporción muy baja de los resultados de la investigación se patenta, por eso es necesario evaluar su potencial de comercialización, ya sea como producto o como proceso (Calderón, 2013; Pérez y Calderón, 2014).
"Es decir, a la idea de patentar tiene que incorporarse un análisis del potencial comercial de las patentes para garantizar cierto éxito en la transferencia tecnológica de las universidades hacia el sector productivo" (García, 2017, p.82, 83).

Así mismo el costo es un punto que lleva a buscar convocatorias de apoyo para subsidiar el pago de una patente. Se sugiere entonces la participación en convocatorias que apoyen en el sentido económico el registro, como la que realiza el Instituto Mexicano de la Propiedad Industrial (IMPI), en coordinación con el Consejo Veracruzano de Investigación Científica Ciencia y Desarrollo Tecnológico (COVEICyDET) para el estado de Veracruz.

Para la convocatoria antes mencionada se debe participar en un taller con una duración de 24 horas, donde el objetivo es adquirir asesoría y conocimiento sobre protección de invenciones, así como para el desarrollo del documento técnico de la descripción de la solicitud de patente o modelo de utilidad. El proceso de manera resumida es el siguiente:

- Búsqueda del nombre apropiado. Considerando la información proporcionada y una búsqueda detallada para verificar que el nuevo registro no sea trate de un plagio.

- $\quad$ Redacción. Se debe realizar el 80\% de la memoria descriptiva de la solicitud de patente del proyecto participante.

- Conclusión. Al término del Taller, dicho borrador será enviado a las personas competentes del IMPI en un plazo aproximado de 15 días hábiles con la siguiente información:

- Título

- $\quad$ Campo técnico

- Antecedentes

- $\quad$ Objeto de la invención

- Descripción de la invención

- $\quad$ Ejemplos

- $\quad$ Reivindicaciones

- $\quad$ Resumen

- Dibujos

- $\quad$ Listado de secuencia

- Depósito de material biológico 
Posteriormente se solicita el pago realizado por COVEICyDET para el ingreso de la solicitud de patente. Se realiza el ingreso ante la delegación de la Secretaría de Economía, con homoclave de formato IMPI xx xxx con fecha de publicación del formato en el DOF 24/05/2018. Las etapas consecuentes son:

Examen de forma. El IMPI en esta etapa tiene hasta tres meses para notificarles si se ha superado el examen de forma, o bien, se les comunica que existe alguna observación que hay que subsanar.

- $\quad$ Etapa en proceso de continuidad. Es la publicación en gaceta de la solicitud de patente, se realizará una vez acreditado el examen de forma, lo antes posible transcurrido 18 meses a la fecha de presentación de la solicitud.

- Examen de fondo. En esta etapa un examinador especializado verificará si la invención es patentable o registrable de acuerdo a los requisitos establecidos en la Ley de la Propiedad Industrial. El instituto podrá emitir un máximo de cuatro requerimientos a efecto de que el solicitante cumpla con las disposiciones legales aplicables. Los registros del modelo de utilidad y patentes otorgados, serán publicados y consultables de forma electrónica y gratuita en la Gaceta del IMPI. Una vez publicados, los expedientes de patentes y registros concedidos, quedarán abiertos a consulta pública en el archivo de patentes y en el visor de documentos de la propiedad industrial (VIDOC) en http://vidoc.impi.gob.mx/.

Etapa de Titulación. Se procederá a la elaboración del título y a la publicación de la patente otorgada.

El proceso de patentamiento es de alrededor de cuatro años.

\section{La Participación de la sociedad}

Después que finaliza la construcción de proyectos ligados a las líneas de investigación del cuerpo académico y se han realizado las pruebas en los prototipos, máquinas y equipos, se buscan las alternativas para que la sociedad los conozca y tengan acceso a ellos, no todos los prototipos, como se mencionó anteriormente, son sujetos de patentamiento, por cuestiones económicas principalmente, aunque se debería realizar este proceso.
En este punto cabe destacar la participación y apoyo de los otros cuerpos académicos de la UTCV, que gracias al convenio formal que se realiza dentro de la institución los integrantes de los otros cuerpos participan activamente en proyectos sociales.

El objetivo principal de los proyectos es su sistematización como una experiencia de trabajo académico desde un enfoque socioformativo (Ambrosio y Hernández, 2018). Cuando el alumno se vuelve consciente de la sociedad en la que participa, surge un reacomodo de ideas para poner en juego sus conocimientos y buscar alternativas en la solución de problemas.

Ambrosio y Hernández (2018) también hacen la referencia a la socialización y comunicación de los resultados mediante la exposición pública de los resultados de la investigación y productos obtenidos hacia la comunidad estudiantil y en general. Por lo que la actividad del cuerpo académico no debiera quedar archivada o verse como una manera de cumplir un horario de trabajo.

La manera que en que se ha hecho llegar los proyectos a la sociedad ha sido a través de los siguientes pasos:

1. Participación en convocatorias del estado y de la región. En este punto han sido favorables las exposiciones de los proyectos porque la gente tiene el primer contacto con la universidad, así como un sin número de especialistas $\mathrm{y}$ representantes de empresas reconocidas.

2. Identificación del grupo de interés o impacto del proyecto. Para que se establezca una relación directa con las personas que puedan beneficiarse con el proyecto, modelo o prototipo.

3. Establecer la relación con el grupo de interés. A través de cursos, talleres y/o pláticas informativas, se deben reunir un número considerable de integrantes para que se pueda replicar o difundir las actividades realizadas dentro de la capacitación.

4. Propiciar y motivar la creación de pequeñas empresas haciendo efectiva la utilización de los proyectos. Con el apoyo de incubadoras en la universidad, cursos para el establecimiento de empresas formales y la búsqueda de recursos públicos y privados.

ARIAS-PRIETO, María Isabel, LARA-SALAZAR, Ignacio Zeferino, RODRIGUEZ-LÓPEZ, Julio Cesar y FERNÁNDEZ-VÁSQUEZ, Celia. La labor de los cuerpos académicos en el programa educativo de Mantenimiento Industrial caso de estudio UTCV. Revista de Gestión Universitaria. 2019 
5. Realizar el seguimiento puntual de las empresas y proyectos. Para buscar mejorar y extender su utilización en más zonas del estado y a nivel nacional.

6. Crear plantas piloto dentro de la universidad. Para incentivar la participación de la sociedad, invitar a estudiantes de diversos niveles educativos y evidenciar los trabajos realizados.

Mato (2018) apunta que las universidades necesitan mejorar su labor en la resolución de necesidades sociales, así como la formación profesional que ofrecen, por lo que es necesario transformar las universidades desde su articulación y compromisos con las sociedades de las cuales forman parte. Es necesario por lo tanto atender la parte de la vinculación entre las universidades y la sociedad, para que se forme un círculo de cooperación y desarrollo en ambos sentidos.

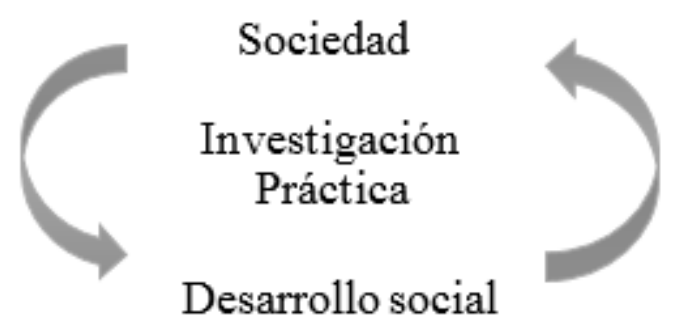

Figura 3 Resultado del vínculo sociedad-investigación

Para Fernández (2017) realizar la vinculación con la sociedad requiere de la investigación técnica y por consiguiente práctica, adquiriendo un carácter flexible y con la facilidad de variar el tema dependiendo de las necesidades de la sociedad. No se puede hablar de tecnología y desarrollo si no se han cubierto las necesidades de la sociedad, si un pueblo no se vuelve partícipe en su propio desarrollo, quedará aislado por sí mismo.

\section{Resultados}

Aunque el número de número de proyectos en la carrera de Ingeniería en Mantenimiento Industrial ha disminuido en el presente año, se espera la participación y desarrollo de nuevos proyectos de impacto social bajo la dirección de los cuerpos académicos del programa educativo y los que integran a la UTCV.
En el periodo mayo-agosto 2019 el cuerpo académico de Gestión de Calidad y Eficiencia Industrial logró reunir la participación de 15 proyectos integradores en el fin de cuatrimestre, de los cuales al menos 5 tienen el potencial para poder desarrollarse y consolidarse para favorecer necesidades de la sociedad. Entre ellos destacan:

- Microgenerador de electricidad. El cual busca por medio de la generación de vapor de una caldera y biogás pretende apoyar la disminución del consumo eléctrico de una casa-habitación promedio de la zona.

- Medidor kW. Hace énfasis en concientizar a la población sobre el uso de la energía eléctrica en los hogares.

- $\quad$ RecyclingP. Fomenta el uso del reciclado de plásticos para generar productos cotidianos.

- Medidor/drone. Proyecto sometido para una participación con el apoyo de investigadores de Estados Unidos y Perú. CutB. Cortador semiautomatizado de botellas de vidrio, pensado para el apoyo de madres solteras y familias de escasos recursos.

El cuerpo académico tuvo la experiencia de iniciar la patente del prototipo participante en el Premio Nacional del Emprendedor, que era la máxima distinción que otorgaba la Secretaría de Economía a través del extinto Instituto Nacional del Emprendedor, siendo finalista junto con cuatro instituciones de prestigio como lo son el Politécnico Nacional, Tecnológico de Monterrey y la Salle, que también presentaron proyectos que resolvían necesidades tanto ambientales como sociales y que por la magnitud de la situación la recomendación fue iniciar el registro ante el IMPI.

En la trayectoria el grupo académico en cuestión, se participa en la convocatoria 2018 propuesta por la universidad que el Instituto Mexicano de la Propiedad Industrial (IMPI), en coordinación con el Consejo Veracruzano de Investigación Científica Ciencia y Desarrollo Tecnológico (COVEICyDET), y el Instituto Tecnológico Superior de Xalapa promueven para apoyo técnico, legal y económico para el registro de patentes, modelos de utilidad y diseños industriales", la cual contempla entre otros aspectos una vez concluido todo el proceso, el pago de los derechos de solicitud de registro ante el IMPI.

ARIAS-PRIETO, María Isabel, LARA-SALAZAR, Ignacio Zeferino, RODRIGUEZ-LÓPEZ, Julio Cesar y FERNÁNDEZ-VÁSQUEZ, Celia. La labor de los cuerpos académicos en el programa educativo de Mantenimiento Industrial caso de estudio UTCV. Revista de Gestión Universitaria. 2019 
Cumpliendo así uno de los objetivos estratégicos plasmados en el Plan Veracruzano de Desarrollo 2016-2018 que consiste en contribuir al desarrollo e innovación tecnológica en el Estado de Veracruz mediante la promoción, vinculación y apoyo a las y los emprendedores, las y los empresarios e instituciones veracruzanas con proyectos de innovación tecnológica.

El 8 de octubre del 2018 se recibió un correo por parte de la Lic. Sandra Cisneros Benítez, Especialista "A" en Propiedad Industrial Oficina Regional Centro, para el envío de documentos y la revisión de estos, y estar en condiciones de ingresar a estudio de solicitud de patente, derivado del taller de redacción de patentes.

El 26 de noviembre del 2018 el biólogo Sergio Gabriel Aguilar Valtierra Coordinador Departamental de Invenciones y Servicios de Información Tecnológica Oficina Regional Centro, adjunta el comprobante de pago que realizó COVEICyDET para el ingreso de solicitud de patente del prototipo mencionado, en el entendido que se terminó la redacción solicitud de patente, conforme a convocatoria. En seguimiento de la redacción de la patente, el 14 de mayo del 2019 se recibe de parte de Román Armenta, analista de planeación del COVEICyDET), un mensaje en el cual se solicita otros documentos de la misma, con la finalidad de tener la información necesaria para buscar los espacios adecuados para la difusión del invento, la patente del cuerpo académico lleva un año ocho meses desde que se inició el registro.

Otras acciones que se realizaron en el año 2018 fue la participación en ferias, exponiendo proyectos que puedan interesar al público en general y al sector industrial, como ejemplo la I Feria de Ciencia y Tecnología en Orizaba, Ver., en la cual se tuvo la participó con dos proyectos. En ese mismo año se participó en el concurso de Expotecnología, organizado por la UTCV, aquí se apoyó la participación de tres proyectos que posteriormente se les daría el seguimiento.

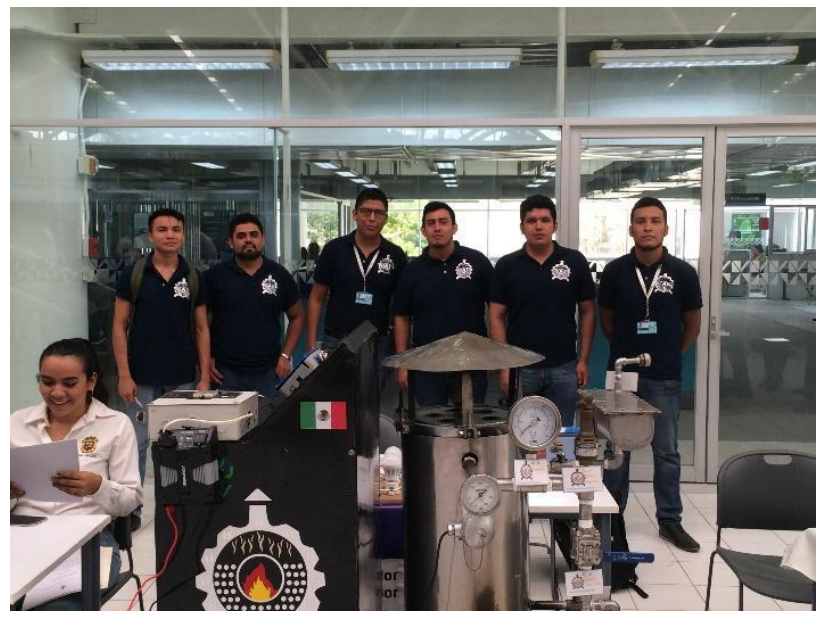

Figura 4 Participación en concursos Fuente: Elaboración Propia

En el mes de julio se asistió al $7 \mathrm{mo}$. Concurso de Creatividad emprendedora organizado por la Universidad Veracruzana en el marco del día del ingeniero. Se obtuvo el segundo lugar con la participación del proyecto "Etiquetadora electroneumática", en ese concurso se asistió con dos proyectos. Dichas participaciones se realizan con proyectos que están en desarrollo y que tienen viabilidad para ser acercados a la sociedad. Cabe subrayar que el interés y la aceptación de los proyectos es siempre positiva, además se toman han tomado en cuenta los comentarios y recomendaciones para la mejora de los proyectos lo que ha brindado experiencias para los alumnos y el cuerpo académico.

En este año se inició una nueva etapa del cuerpo académico y los integrantes se dieron a la tarea de iniciar la impartición de talleres con los prototipos creados, en primera instancia fue la impartición de talleres a grupos de mujeres en el DIF de Córdoba, con el taller "Corte de botellas de vidrio mediante máquina semiautomatizada", causando mucho interés a los grupos de mujeres, en el cual a través del uso del equipo y promoviendo un modelo de negocio para la compra del equipo, las participantes realizaron diferentes diseños para la comercialización de producto como vasos, lámparas, veladoras, etc.

La convocatoria fue dirigida a través del DIF de Córdoba Ver., donde se especificaron los requisitos para el taller. Durante la impartición del taller se mostró la máquina, se explicó el diseño y uso, así como el trabajo que realiza el cuerpo académico para impulsar a jóvenes en el diseño y construcción de proyectos que ayuden a resolver problemas del entorno. 


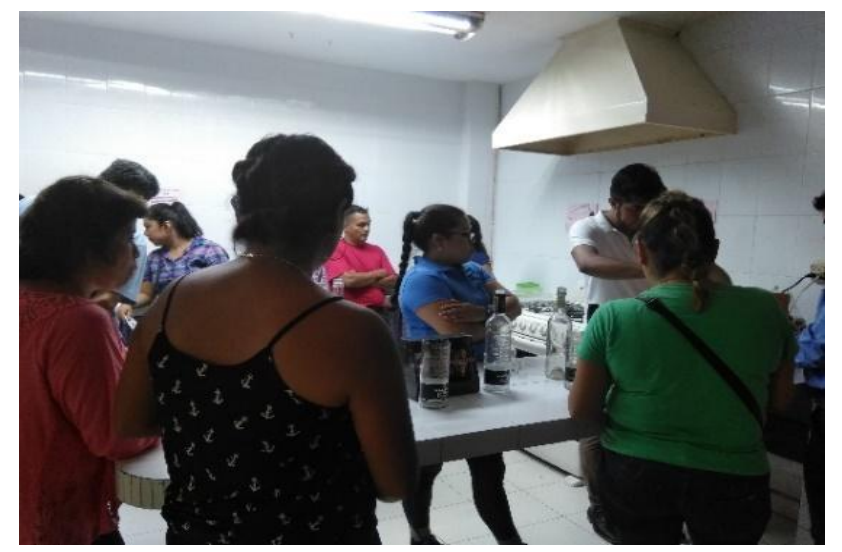

Figura 5 Participación ciudadana en los proyectos Fuente: Elaboración Propia

Para finalizar, el cuerpo académico se ha fijado la meta para inicios del 2020, en la construcción de una planta piloto para el reciclado de PET, con este proyecto se demostrarán los prototipos que se han generado hasta la fecha en cuestión de reciclado de plástico, se plantea difundirla con las escuelas de la región e invitar a la ciudadanía para que puedan copiar el modelo y poder iniciar sus propias empresas, el cuerpo académico facilitará en la medida posible las herramientas necesarias para replicar los proyectos y poder establecer más plantas que apoyen la economía familiar y se busque la creación de empleos directos e indirectos.

\section{Conclusiones}

El camino que debe recorrer un cuerpo académico nuevo es sin duda un proceso que se va descubriendo con el apoyo y las propuestas de los integrantes y de las academias que conforman el ámbito de la investigación, en las universidades se debería dar prioridad a la labor que éstos desempeñan. Es necesario reconocer la importancia de la investigación pues a través de ésta se vincula a las demandas sociales y juega un papel protagonista, superando el papel académico-profesionalizante que suele adquirir $\mathrm{y}$ transformando a las universidades en universidades de primera línea (Cazorla, 2017).

Los resultados que se han venido dando han sido gracias a la implementación y seguimiento de los proyectos integradores que se realizan en la UTCV, propiciando los medios idóneos para la investigación práctica, entendiéndose esta última como la relación directa del conocimiento con la solución de los problemas del contexto social.
Con la numerosa participación de prototipos de en la carrera de Mantenimiento Industrial se vuelve factible el buscar el patentamiento de prototipos, por lo que es necesario realizar un análisis más técnico de los mismos, ya que en la actualidad existen muchos equipos que realizan la misma función para una tarea determinada, sin embargo, si se logra detectar lo que resuelve el equipo de manera técnica, se puede iniciar el proceso de patente y proteger las ideas de los inventores para evitar el plagio.

El principal reto del cuerpo académico de Gestión de Calidad y Eficiencia Industrial, ha sido acercar a la gente el desarrollo tecnológico que se hace a través de los proyectos integradores de los estudiantes, la visión a futuro es sin duda continuar desarrollando proyectos, máquinas y prototipos en beneficio de la sociedad y procurando en todo momento salvaguardar el medioambiente y aprovechar de manera eficiente el uso de materiales y recursos naturales. Esto se puede hacer posible por medio de las convocatorias, participación en eventos tecnológicos y la puesta en marcha de plantas piloto, los cuales servirán de ejemplo para los más jóvenes, y a través de estos mecanismos se demuestre de manera evidente los alcances que se pueden lograr, en vistas de la conformación de empresas, generación de empleos y mayor oportunidad de desarrollo económico para las personas y las organizaciones interesadas.

\section{Agradecimiento}

Agradecemos el financiamiento por parte de la Universidad Tecnológica del Centro de Veracruz para la presentación del artículo, en especial al área de Investigación por el apoyo brindado estos años de trabajo.

\section{Referencias}

Acevedo, B. y Severiche, C. (2016). Estrategia de investigación formativa en educación tecnológica: el caso del Proyecto Integrador. Itinerario Educativo. 67, 103-121. DOI: 30. 10.21500/01212753.2891.

Ambrosio, R. y Hernández, J. (2018). Aprendizaje por proyectos, una experiencia socioformativa. Voces De La Educación, 3(5), 3-19. Recuperado a partir de https://www.revista.vocesdelaeducacion.com.m x/index.php/voces/article/view/88 
Beltrán, A., Sevilla, D., Martín. J. (2018). Los cuerpos académicos: creencias y percepciones de profesores asociados. Investigación Cualitativa, 3(1), 58-72.

Calderón, G. (2013). La gestión de patentes universitarias. El caso de la unam. México: unam-dgei

Cazorla, A. Hacia una universidad de investigación desde una profesional: estrategias. En A. Cazorla (Ed.), La universidad: motor de transformación de la sociedad (16-30). Madrid, España: Universidad Politécnica de Madrid (UPM).

Fernández, C. (2017). La vinculación con la sociedad en las universidades de Investigación. En A. Cazorla (Ed.), La universidad: motor de transformación de la sociedad (52-61). Madrid, España: Universidad Politécnica de Madrid (UPM).

García, R. (2017). Patentamiento universitario e innovación en México, país en desarrollo: teoría y política. doi.org/10.1016/j.resu.2017.11.001

López Barrios, Janet (2008). Reseña de "Las universidades tecnológicas mexicanas. Un modelo eficaz, una inversión pública exitosa, un sistema a fortalecer" de Jacques Mazeran. Tiempo de Educar, 9(17), 147-151.

Mato, D. (2018). Repensar y transformar las universidades desde su articulación y compromiso con las sociedades de las que forman parte. +E: Revista de Extensión Universitaria, 8(9), julio-diciembre, 38-52. doi: 10.14409/extension.v8i9.Jul-Dic.7837.

Pérez, P. y Calderón, G. (2014). Análisis de los procesos de comercialización de tecnología en dos instituciones de educación superior mexicanas. Journal of Technology Management \& Innovation, 9(3), 196-209.

Regata, J. y Barajas, A. (2019). Funciones y niveles de desempeño requeridos a estudiantes de Ingeniería Industrial según género y tipo de empresa. Revista Educación en Ingeniería, 14(28), 88- 94. 